\title{
THE FREQUENCY OF BARRED SPIRAL GALAXIES IN THE NEAR-INFRARED ${ }^{1}$
}

\author{
Paul B. Eskridge, ${ }^{2}$ Jay A. Frogel, ${ }^{2,3}$ Richard W. Pogge, ${ }^{2}$ Alice C. Quillen, ${ }^{3,4}$ Roger L. Davies, ${ }^{5}$ D. L. DePoy, ${ }^{2,3}$ \\ Mark L. Houdashelt, ${ }^{6}$ Leslie E. Kuchinski, ${ }^{3,7}$ Solange V. Ramírez, ${ }^{2,3}$ K. Sellgren, ${ }^{2,3}$ \\ Donald M. Terndrup, ${ }^{2,3}$ AND GlenN P. TIEDE ${ }^{3,8}$ \\ Received 1999 July 15 ; accepted 1999 October 26
}

\begin{abstract}
We have determined the fraction of barred galaxies in the $H$-band for a statistically well-defined sample of 186 spirals drawn from the Ohio State University Bright Spiral Galaxy Survey. We find 56\% of our sample to be strongly barred in the $H$ band while another $16 \%$ is weakly barred. Only $27 \%$ of our sample is unbarred in the near-infrared. The RC3 and the Carnegie Atlas of Galaxies both classify only about $30 \%$ of our sample as strongly barred. Thus strong bars are nearly twice as prevalent in the near-infrared as in the optical. The frequency of genuine optically hidden bars is significant but lower than many claims in the literature: $40 \%$ of the galaxies in our sample that are classified as unbarred in the RC3 show evidence for a bar in the $H$ band while the Carnegie Atlas lists this fraction as $66 \%$. Our data reveal no significant trend in bar fraction as a function of morphology in either the optical or $H$ band. Optical surveys of high-redshift galaxies may be strongly biased against finding bars, as bars are increasingly difficult to detect at bluer rest wavelengths.
\end{abstract}

Key words: galaxies: fundamental parameters — galaxies: spiral — galaxies: statistics — galaxies: structure

\section{INTRODUCTION}

Roughly $30 \%$ of spiral galaxies are strongly barred in the optical (de Vaucouleurs 1963) while another $25 \%$ are weakly barred (e.g., Sellwood \& Wilkinson 1993). Evidence that bars in spiral galaxies are more obvious in the nearinfrared (NIR) than the visible goes back to Hackwell \& Schweizer (1983), who noted that the weak optical bar in NGC 1566 was a strong feature in the $H$ band, with "azimuthal brightness variations of up to $\sim 25 \%$." Their main conclusion was that these brightness variations reflected true mass variations. The case for this interpretation is reviewed by Frogel, Quillen, \& Pogge (1996). Briefly, the main reasons are that the $H$-band light is sampling the dominant, old stellar population and is relatively unaffected by the presence of bright young blue stars or by dust, both of which strongly influence optical images. Since Hackwell and Schweizer's initial discovery, several other examples of NIR bars that are less obvious in the optical have been reported (e.g., Thronson et al. 1989-NGC 1068; Block \& Wainscoat 1991-NGC 309; Block et al. 1994-NGC 4736; Mulchaey \& Regan 1997; Seigar \& James 1998; Knapen, Shlosman, \& Peletier 2000). While useful in pointing out specific cases of optically hidden bars, none of these

\footnotetext{
${ }^{1}$ Based partially on observations obtained at the Cerro Tololo InterAmerican Observatory, operated by the Association of Universities for Research in Astronomy, Inc., under cooperative agreement with the National Science Foundation.

${ }^{2}$ Department of Astronomy, Ohio State University, Columbus, $\mathrm{OH}$ 43210; eskridge@astronomy.ohio-state.edu; frogel@astronomy.ohiostate.edu.

${ }^{3}$ Visiting Astronomer, Cerro Tololo Inter-American Observatory.

${ }^{4}$ Steward Observatory, University of Arizona, Tucson, AZ 85721.

${ }^{5}$ Department of Physics, University of Durham, South Road, Durham DH1 3LE, England, UK.

${ }^{6}$ Department of Physics and Astronomy, Johns Hopkins University, Baltimore, MD 21218.

${ }^{7}$ NASA/IPAC, California Institute of Technology, Mail Stop 100-22, Pasadena, CA 91125.

${ }^{8}$ National Optical Astronomy Observatories, Tucson, AZ 85719.
}

NIR studies are based on large, statistically well-defined samples of galaxies and so cannot address the following fundamental question: What fraction of spiral galaxies have bars?

A determination of the true frequency of bars and other nonaxisymmetric structures is important for a number of reasons. Bars can initiate spiral density waves (e.g., Toomre 1969; Kormendy 1982a) and stellar rings (Buta 1986). Stellar bars may also provide a means to channel gas into nuclear regions of galaxies by inducing gravitational torques on gas trailing the bar, thus robbing the gas of angular momentum and driving it inward to fuel nuclear starbursts or active galactic nuclei (e.g., Kormendy 1982b; Shlosman, Frank, \& Begelman 1989; Pfenniger \& Norman 1990; Quillen et al. 1995; Piner, Stone, \& Teuben 1995). Finally, bars may be important for driving the secular evolution of bulges, both by triggering nuclear starbursts and by kinematic heating of the inner disk (Pfenniger \& Norman 1990; Combes et al. 1990).

A number of studies indicate that strongly barred galaxies must have maximal (or nearly maximal) disks. Debattista \& Sellwood (1998) find that the absence of slow bars requires maximum disks, which transfer very little angular momentum to the low density halos. Also, bars are often associated with rings (e.g., Buta 1995). Such bar/ring structures appear to require a maximal disk for stability (Quillen $\&$ Frogel 1997). Hydrodynamic simulations often find that the gas dynamics in barred systems require massive bars (e.g., Lindblad, Lindblad, \& Athanassoula 1996; Weiner et al. 1996). The situation is strikingly different for galaxies that are optically unbarred. Courteau \& Rix (1999) find that the Tully-Fisher relationship shows no dependence on disk scale length for a sample of optically unbarred galaxies and conclude from this that disks are massless. Bottema (1993) studied the velocity dispersion profiles of a sample of optically unbarred galaxies and concluded that these galaxies are all submaximal. To make matters more puzzling, there does not appear to be any difference in the average rotation curves of optically barred and unbarred galaxies (Bosma 
1996). Thus a determination of the true frequency of bars (hereafter "bar fraction") in spiral galaxies is essential for understanding the so-called dark matter problem and, more generally, the structure of spiral galaxy halos.

In this paper, we present the first statistical study of the frequency of bars in the NIR based on the Ohio State University (OSU) Bright Spiral Galaxy Survey. In $\S 2$, we briefly describe the survey. We examine the NIR bar fraction of our sample in $\S 3$, and we compare this to the results for the same sample from standard optical catalogs. Our sample is large enough that we can study the effect of morphology on bar statistics in $\S 4$. In $\S 5$, we discuss some implications of our results, consider the possibility of various biases in our analysis, and indicate promising areas for future research.

\section{OSU BRIGHT SPIRAL GALAXY SURVEY}

The OSU Bright Spiral Galaxy Survey deep, photometrically calibrated $B V R J H K$ images of a magnitudelimited sample of 205 spirals with $T \geq 0, B \leq 12$, and $D \leq 6^{\prime}$ in de Vaucouleurs et al. (1991, hereafter RC3). A full description of the survey, including the selection criteria and observational strategy, will be presented in Eskridge et al. (2000). Because this is a large, clearly defined sample of well-resolved galaxies, it is especially useful for addressing the statistical properties of spiral galaxies.

Although the data from the OSU Survey are not yet fully photometrically calibrated, they are well suited for performing the first statistical analysis of the presence of bars in NIR images of spiral galaxies. We base our analysis on our $H$-band images, as they generally have higher signal-tonoise ratios than do our $K$-band images and are nearly as unaffected by dust extinction. For this paper, we consider only galaxies that do not display gross peculiarities and do not have close companions of similar (or greater) luminosity. This gives us a total sample of 186 galaxies.

\section{BAR FREQUENCY IN THE NEAR-INFRARED AND OPTICAL}

As our sample is selected from the RC3, we can compare our $H$-band bar classifications with the RC3 optical bar classifications for all 186 objects in our sample. We also compare our $H$-band bar classifications with the optical classifications of Sandage \& Bedke (1994; hereafter CAG) for the 166 objects in common with our sample. We present the statistics of our classifications in Table 1. The two optical catalogs differ with respect to the classification of weak bars, representing the extremes of approach to the issue. The CAG generally classifies a galaxy as unbarred unless the presence of a bar is very clear, whereas the RC3 tends to classify galaxies as weakly barred if there is even the slightest evidence for a bar.

\subsection{Bar Frequency in the $H$ Band}

We inspected the $H$-band images of the 186 galaxies in our sample and assigned a Hubble type to each. We used the standard, subjective classification criteria described in the CAG and the RC3. We made these classifications with no prior knowledge of the optical classifications of the sample (except, of course, for the Survey definition). As this paper is concerned with bar frequency only, we defer a full discussion of our classification scheme to Eskridge et al. (2000). We adopt the RC3 distinction between strongly barred (SB), weakly barred (SAB), and unbarred (SA) galaxies, although it is clear from the results presented below that we were more conservative in our assignment of galaxies as weakly barred than is the RC3. We note that the RC3 classification of weakly barred (SAB) differs from the CAG classification of intermediate barred (S/SB). As the terms "barred," "unbarred," "weakly barred," and "intermediate barred" are not explicitly defined in the optical catalogs, we provide examples in Figure 1 of $B$-band and $H$-band images of three galaxies: NGC 3223 (unbarred in the $H$ band, the RC3, and the CAG); NGC 1371 (weakly barred in the $H$ band and in the RC3, unbarred in the CAG); NGC 613 (strongly barred in the $H$ band, in the $\mathrm{RC} 3$, and in the CAG).

One of us (P. B. E.) classified the entire sample twice, with excellent overall agreement between the two trials: more than $80 \%$ of the classifications agreed to within two subtypes (i.e., Sa to $\mathrm{Sb}$ ). We also cross-checked our results by comparing classifications done by two of us (P. B. E. and J. A. F.) for 40 galaxies. Again, more than $80 \%$ of the tested galaxies were assigned types that agreed to within two subtypes. This compares well with the scatter between classifiers reported by Naim et al. (1995). We note that we do not explicitly consider the scale of the bar when making these classifications; galaxies with short, high-contrast bars are considered barred. As the optical catalogs are based on photographic images, there is some chance of a bias against finding short, high-contrast bars in these works. We discuss this point in more detail in $\S 3.2$.

\subsection{Comparison with Optical Bar Types}

The bar classification from the RC3 is given for our sample in Table 1 . The left-hand panel of Figure 2 shows the distribution of our sample in a $3 \times 3$ matrix of the RC3

TABLE 1

Bar Fraction in the $H$ Band and Optical Catalogs

\begin{tabular}{|c|c|c|c|c|c|c|}
\hline \multirow[b]{2}{*}{$\begin{array}{l}\text { BAR ClasS } \\
\text { (1) }\end{array}$} & \multicolumn{2}{|c|}{$H$ BAND } & \multicolumn{2}{|c|}{$\mathrm{RC} 3$} & \multicolumn{2}{|c|}{ CAG } \\
\hline & $\begin{array}{c}\text { Fraction }(\%) \\
\text { (2) }\end{array}$ & $\begin{array}{c}\text { Number } \\
\text { (3) }\end{array}$ & $\begin{array}{c}\text { Fraction }(\%) \\
\text { (4) }\end{array}$ & $\begin{array}{c}\text { Number } \\
(5)\end{array}$ & $\begin{array}{c}\text { Fraction }(\%) \\
(6)\end{array}$ & $\begin{array}{c}\text { Number } \\
\text { (7) }\end{array}$ \\
\hline SB $\ldots \ldots \ldots \ldots \ldots$ & 56 & 105 & 35 & 65 & 27 & 44 \\
\hline SAB ........... & 16 & 30 & 30 & 56 & 4 & 6 \\
\hline $\mathrm{SB}+\mathrm{SAB} \ldots \ldots$ & 73 & 135 & 65 & 121 & 30 & 50 \\
\hline SA $\ldots \ldots \ldots \ldots$ & 27 & 51 & 35 & 65 & 70 & 116 \\
\hline $\mathrm{SA}+\mathrm{SAB} \ldots \ldots$ & 44 & 81 & 66 & 121 & 74 & 122 \\
\hline
\end{tabular}

NoTE.-In columns 2, 4, and 6 we give the fraction of galaxies in our sample with a given bar class in the $H$ band, the RC3, and the CAG, respectively. Columns 3, 5 , and 7 give the total number of galaxies in our sample in each bar class from the same sources. 




FIG. $1 a$

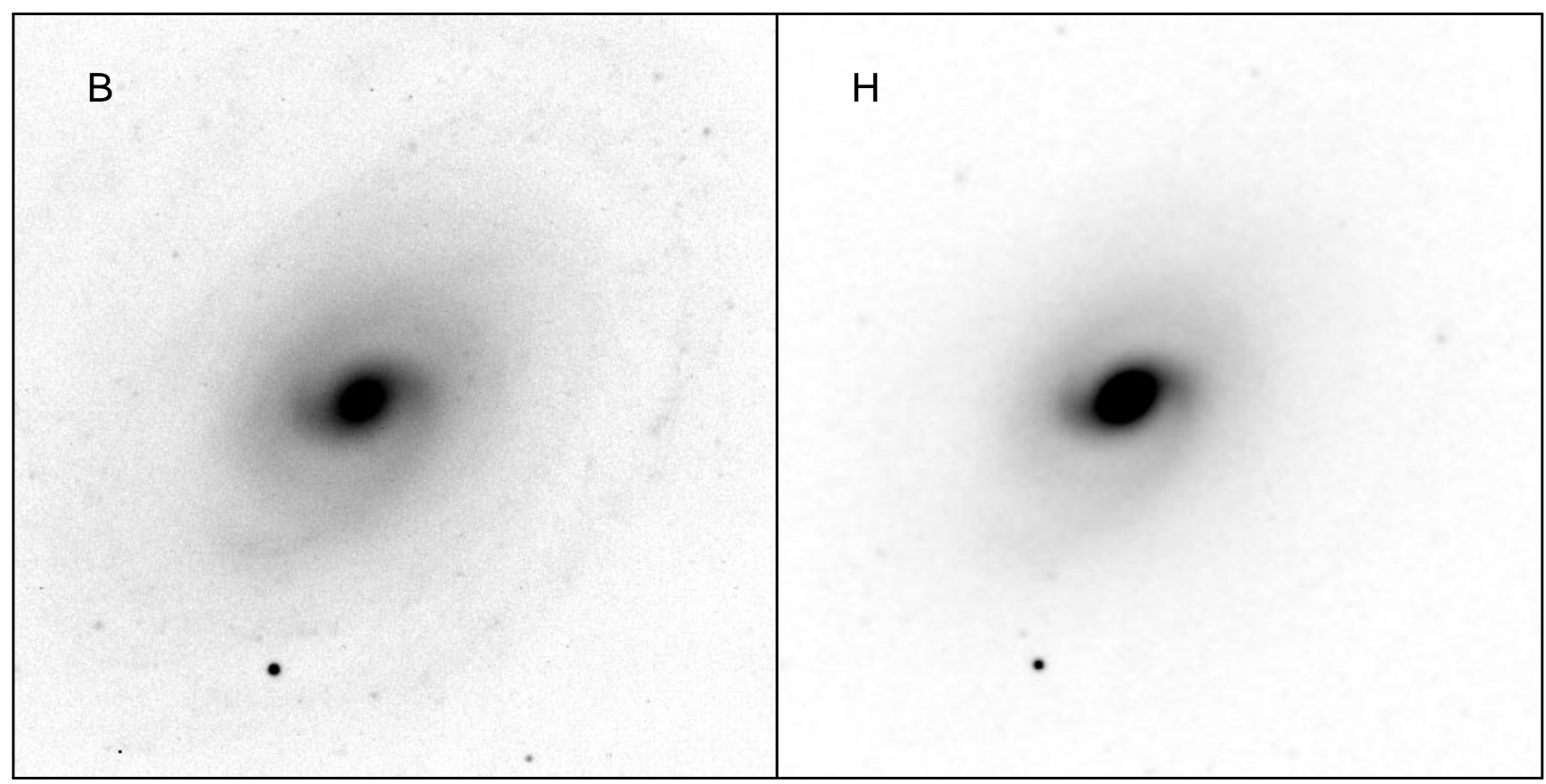

FIG. $1 b$

FIG. 1.- $B$-band and $H$-band images of (a) NGC 3223 (unbarred in the $H$ band, in the RC3, and in the CAG); (b) NGC 1371 (weakly barred in the $H$ band and in the RC3, unbarred in the CAG); and (c) NGC 613 (strongly barred in the $H$ band, in the RC3, and in the CAG).

versus our $H$-band bar classification. We classify relatively few galaxies as weakly barred compared with the RC3; bars are typically detected in the optical if they are present, but they appear stronger in the NIR. Of the 105 galaxies we classify as strongly barred in the $H$ band, there are 10 that are classified as unbarred (SA) in the RC3. We list these galaxies in Table 2. Most often, these are systems with relatively short, high-contrast $H$-band bars that are hidden in the optical by patchy foreground extinction and complex inner arm structure. In Figure 3 we show $B$ band and
$H$-band images of two galaxies with optically hidden bars, NGC 5161 and NGC 5085. In $\S 4$ we shall revisit the topic of optically hidden bars in the context of galaxy morphology.

There are 15 galaxies, listed in Table 3, that have stronger optical bar-types in the RC3 than we assign to the $H$-band images (see Fig. 2). We reinspected our $H$-band images of these galaxies, as well as both the CAG and our own optical CCD images. Of these 15 galaxies, three are low surfacebrightness irregular galaxies (NGC 625, ESO 383-G87, and 




FIG. 1c

NGC 7713) that have vaguely rectangular isophotes. While barred irregular galaxies certainly do exist (e.g., the LMC and NGC 6822), we see no evidence for a bar in any of these three objects in either the optical or the NIR. Three galaxies (NGC 1421, A0908-08, and IC 5052) are edge-on spirals. It is notoriously difficult to detect bars in edge-on galaxies based on imaging data alone (see Kuijken \& Merrifield 1995), thus we are not troubled by the disagreement for these galaxies. Three of the 15 galaxies are classified as strongly barred in the RC3, but we classify them as weakly barred in the $H$-band. Five of the remaining six are classified as weakly barred in the RC3 and as unbarred by us.
The final galaxy (NGC 4856) is strongly barred in the RC3 and unbarred in our classification. We do not see evidence for bars in our deep optical CCD images for these last six galaxies. We speculate that these galaxies may have been misclassified in the RC3 because of the limitations of the photographic images available for classification and note that none of these galaxies are classified as barred in the CAG.

We also compare our bar classification with the optical classification from CAG for the 166 galaxies we have in common (see Table 1). A large number of galaxies classified as optically unbarred in the CAG show bars in the NIR.

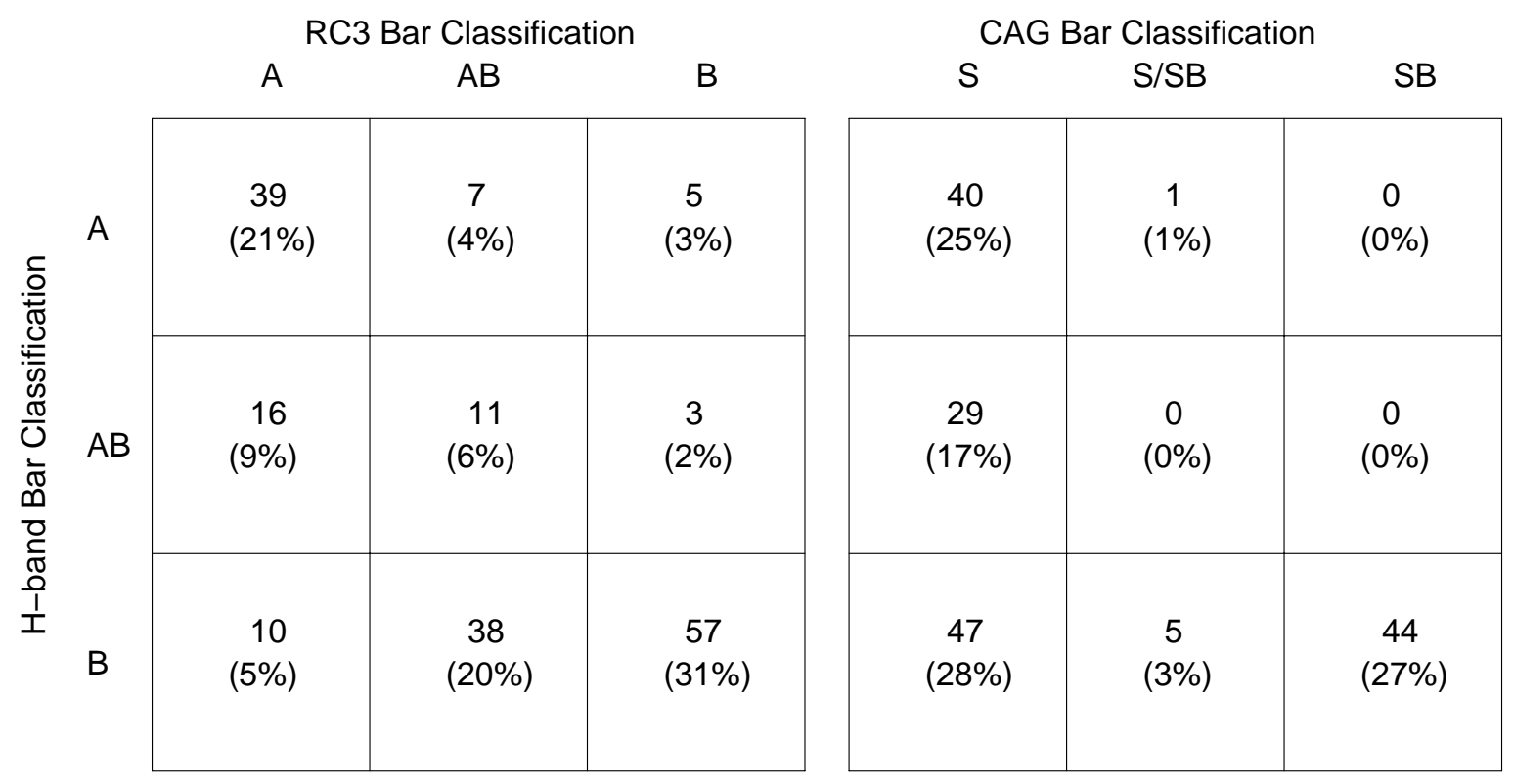

FIG. 2.-Grids showing the distribution of galaxies in cells according to the RC3 and our $H$-band bar classification (left-hand panel) and according to the $\mathrm{CAG}$ and our $\mathrm{H}$-band bar classification (right-hand panel). 
TABLE 2

STRONGLy INFRARED-BARRED GALAXIES, UNBARRED IN RC3

\begin{tabular}{ccl}
\hline \hline Galaxy & H-band Type & RC3 Type \\
\hline NGC $1511 \ldots \ldots$ & SBd? & SAa: pec \\
NGC $2280 \ldots \ldots$ & SBbc & SA(s)cd \\
NGC $3675 \ldots \ldots$ & SB(r)a & SA(s)b \\
NGC $4388 \ldots \ldots$ & SB(r)a & SA(s)b: sp \\
NGC $4450 \ldots \ldots$ & SBab & SA(s)ab \\
NGC $4504 \ldots \ldots$ & SBcd & SA(s)cd \\
NGC $5161 \ldots \ldots$ & SBab & SA(s)c: \\
NGC $5483 \ldots \ldots$ & SBc & SA(s)c \\
NGC $5962 \ldots \ldots$ & SBab & SA(r)c \\
NGC $6902 \ldots \ldots$ & SB(r)a & SA(r)b \\
\hline
\end{tabular}

The right-hand panel of Figure 2 shows the distribution of our sample in a $3 \times 3$ matrix of CAG versus $H$-band bar classification. We note that there is only one object classified as intermediate (S/SB) in the CAG that we classify as unbarred in the $H$ band: IC 5052, noted above. There are no galaxies classified as strongly barred in the CAG that we do not also classify as strongly barred in the $H$ band.

One potential bias in the optical classifications is the possibility that bars are undetected in the optical because of the use of photographic images for classification in the RC3 and CAG. In many of these images, the central parts of the galaxy are burned out. Thus, if there were a small-scale nuclear bar it might have escaped detection by the optical classifiers. We checked this possibility by reexamining our $H$-band images and the optical images in the CAG for all galaxies that we classified as barred or weakly barred in the $H$ band but that were classified as unbarred in either the $\mathrm{RC} 3$ or the CAG. There are 10 galaxies, listed in Table 4, that could suffer from this dynamic range problem. Of these, only one (NGC 2280) is classified as unbarred in both the RC3 and CAG. The other nine are classified as either SB or SAB in the RC3. For all 10 galaxies, we then examined our OSU Survey $B$-band images to see if the $H$-band bar was detectable in high dynamic range optical data. In five of

TABLE 3

GalaXIES WITH Stronger RC3 Bars than $H$-BAND Bars

\begin{tabular}{lcc}
\hline \hline$H$-band SA, RC3 SAB & $H$-BAND SA, RC3 SB & $H$-band SAB, RC3 SB \\
\hline NGC $157 \ldots \ldots \ldots \ldots \ldots$ & NGC 625 & NGC 1617 \\
NGC $278 \ldots \ldots \ldots \ldots \ldots$ & NGC 4856 & NGC 1703 \\
NGC $1421 \ldots \ldots \ldots \ldots$. & ESO $383-$ G87 & NGC 7412 \\
NGC $1964 \ldots \ldots \ldots \ldots$. & IC 5052 & $\ldots$ \\
A0908-08 $\ldots \ldots \ldots \ldots \ldots$ & NGC 7713 & $\ldots$ \\
NGC $4580 \ldots \ldots \ldots \ldots$ & $\ldots$ & $\ldots$ \\
NGC $5248 \ldots \ldots \ldots \ldots$ & $\ldots$ & $\ldots$ \\
\hline
\end{tabular}

TABLE 4

INFRARED-BARRED GaLAXIES WITH PoteNTIAL Optical Dynamic Range Problems

\begin{tabular}{lc}
\hline \hline Unbarred in $B$-band CCD & Barred in $B$-band CCD \\
\hline NGC $779 \ldots \ldots \ldots \ldots \ldots \ldots$. & NGC 1317 \\
NGC $2280 \ldots \ldots \ldots \ldots \ldots \ldots$. & NGC 3893 \\
NGC $3166 \ldots \ldots \ldots \ldots \ldots \ldots$. & NGC 4448 \\
IC $4444 \ldots \ldots \ldots \ldots \ldots \ldots$. & NGC 4699 \\
IC $5325 \ldots \ldots \ldots \ldots \ldots \ldots$. & NGC 4781 \\
\hline
\end{tabular}

the 10 galaxies, no bar is visible in our deep $B$-band images. In two cases, the bar is clearly visible (NGC 4448, NGC 4699). In two further cases (NGC 3893, NGC 4781) the bar is visible, but we would not consider the galaxy to be barred without the prior knowledge of the $H$-band bar. The remaining galaxy is NGC 1317, which has both a nuclear bar and a large-scale bar. The nuclear bar is weakly visible in our $B$-band image. Thus we have a few examples of galaxies that are classified as unbarred in the CAG (but not in the RC3) that could be misclassified because of the limited dynamic range of the optical plates. We conclude that misclassification of galaxies due to the poor dynamic range of the optical classifying plates is not a serious bias for our current study.

\section{BAR FRACTION AS A FUNCTION OF MORPHOLOGY}

We have a large enough sample that we are able to examine the bar fraction as a function of morphology. In Figure 4 we plot the bar fraction as a function of morphological type from the RC3 (Fig. 4a), the CAG (Fig. 4b), and our $H$-band classification (Fig. 4c). In the $H$ band, roughly two-thirds of the sample show bars, with no significant dependence on morphology. In the optical, both the RC3 and the CAG have a larger bar fraction for the earliest spiral types compared with the intermediate types. This is not statistically significant because of the small number of galaxies in the earliest type bin for both the RC3 and CAG. However, if one adopts the hypothesis that bars are structures in the old stellar population, such an optical excess in the earliest types would be expected because of the relative lack of dust and young stars in early-type spirals compared with that of later types. The difference between the RC3 and CAG in the latest types can be ascribed entirely to the small number of very late-type systems in our sample according to the CAG. The RC3 and CAG agree that roughly onethird of intermediate spirals (Sa through Scd) have strong bars. In the $H$-band, however, about $54 \%$ of intermediate spirals have strong bars.

Because the RC3 and the CAG treat weak bars so differently, we examine the influence of morphology on the issue of optically hidden bars in two ways. First, we examine the fraction of unbarred galaxies (SA) in the two optical catalogs that we find to have $H$-band bars (SB and $\mathrm{SAB}$ ). Second, we examine the fraction of galaxies that are not strongly barred (SA and SAB) in the two optical catalogs that we find to be strongly barred (SB) in the $H$ band. In Figure 5, we plot the fraction of optically unbarred galaxies that have bars in the $H$ band, as a function of morphology, for the two optical catalogs. The solid lines show the fraction of strong $H$-band bars while the dashed lines include weak $H$-band bars. When we compare with the RC3 (Fig. $5 a$ ), we find that roughly half of optically unbarred spiral galaxies (types $\mathrm{Sa}-\mathrm{Scd}$ ) reveal the presence of a bar in the $\mathrm{H}$-band. The fraction increases toward later types. As noted earlier, this is most likely because of the increasing obscuration caused by dust and the increasing prominence of young population tracers in the later type spirals. Comparing, instead, with the CAG (Fig. 5b), we find a larger fraction of hidden bars and no evidence for a trend with morphological type. About two-thirds of the optically unbarred galaxies with types between $\mathrm{Sa}$ and $\mathrm{Scd}$ in the CAG have bars in the $H$ band. In Figure 6 we plot the fraction of galaxies with strong $H$-band bars that are not classified as strongly barred in the optical. Here the solid lines show the 


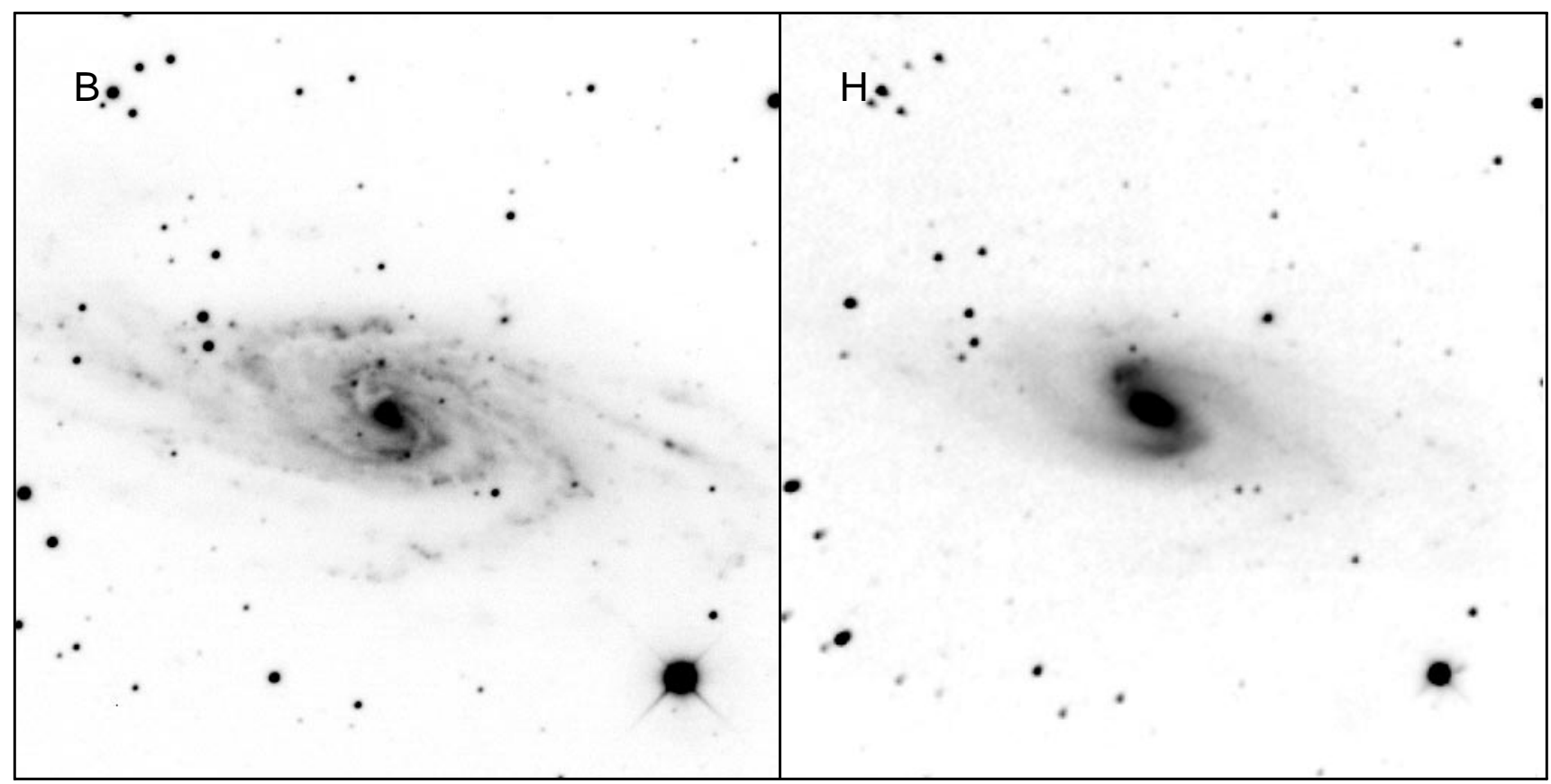

FIG. $3 a$

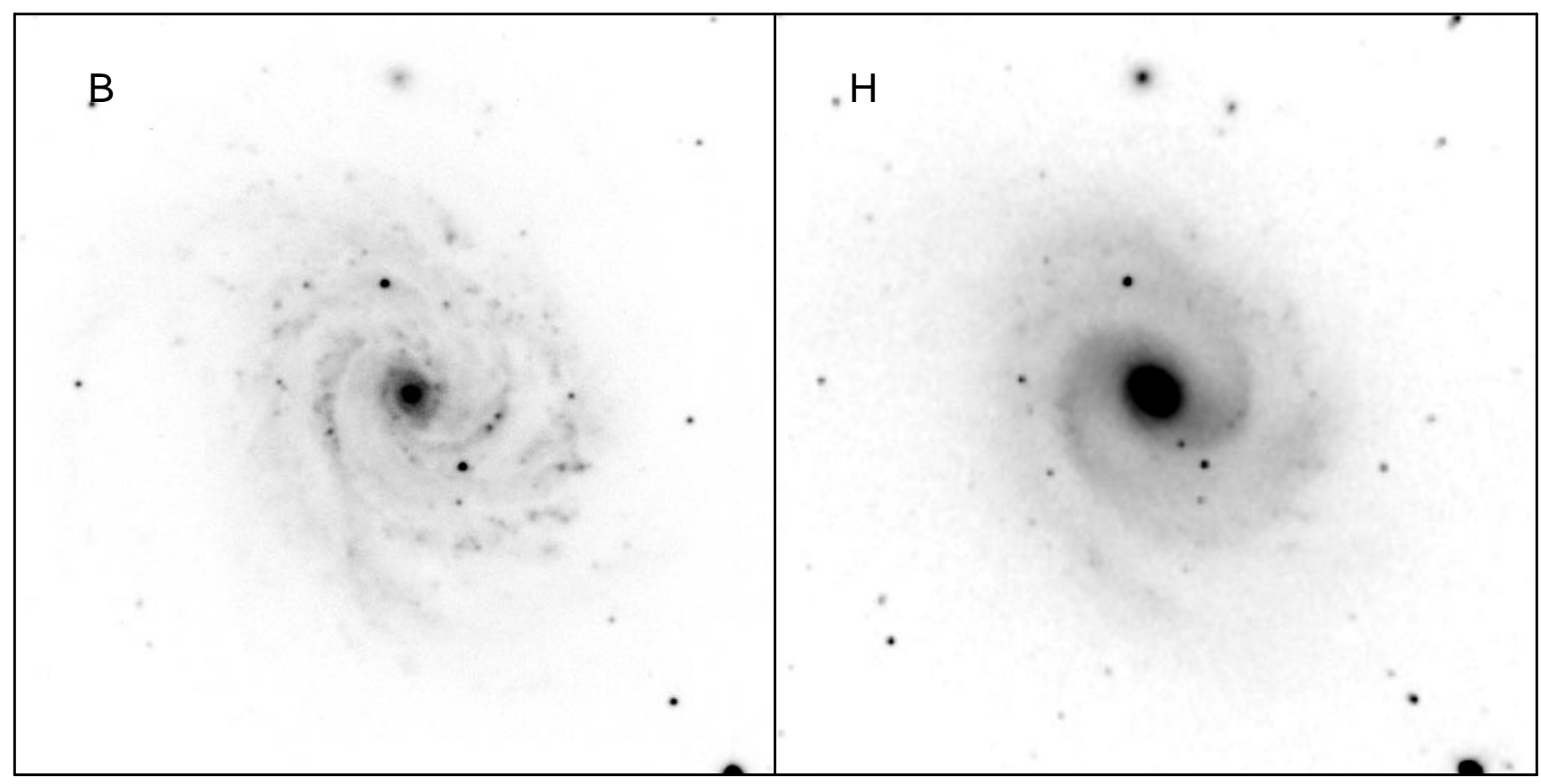

FIG. $3 b$

FIG. 3. - B-band and $H$-band images of $(a)$ NGC 5161 and (b) NGC 5085. These are galaxies classified as optically unbarred (SA) in the RC3 but as barred (SB and SAB, respectively) in the $H$ band by us.

results including only the optically unbarred galaxies while the dashed lines include the optically weakly barred galaxies. When we compare with the RC3 (Fig. 6a), we find that roughly $40 \%$ of spirals without a strong optical bar reveal a strong bar in the $H$ band, with no strong evidence for morphological dependence. Comparing with the CAG (Fig. 6b), we find essentially the same result.

\section{SUMMARY AND DISCUSSION}

We have performed the first analysis of the bar fraction in the NIR ( $H$ band) of a large (186 galaxies) statistically well- defined sample of spiral galaxies drawn from the OSU Bright Spiral Galaxy Survey. The central result of this work is that the fraction of strongly barred galaxies is much higher in the NIR (nearly $60 \%$ of our sample) than previously found in the optical (roughly $30 \%$ of our sample). In detail, we find $56 \%$ of the sample (105 galaxies) to be strongly barred in the $H$ band and $72 \%$ (134 galaxies) to be barred at some level. We note that this agrees well with the results of Mulchaey \& Regan (1997) and Knapen et al. (2000) for much smaller samples. For the same sample of objects, the RC3 finds $34 \%$ to be strongly barred and $64 \%$ 


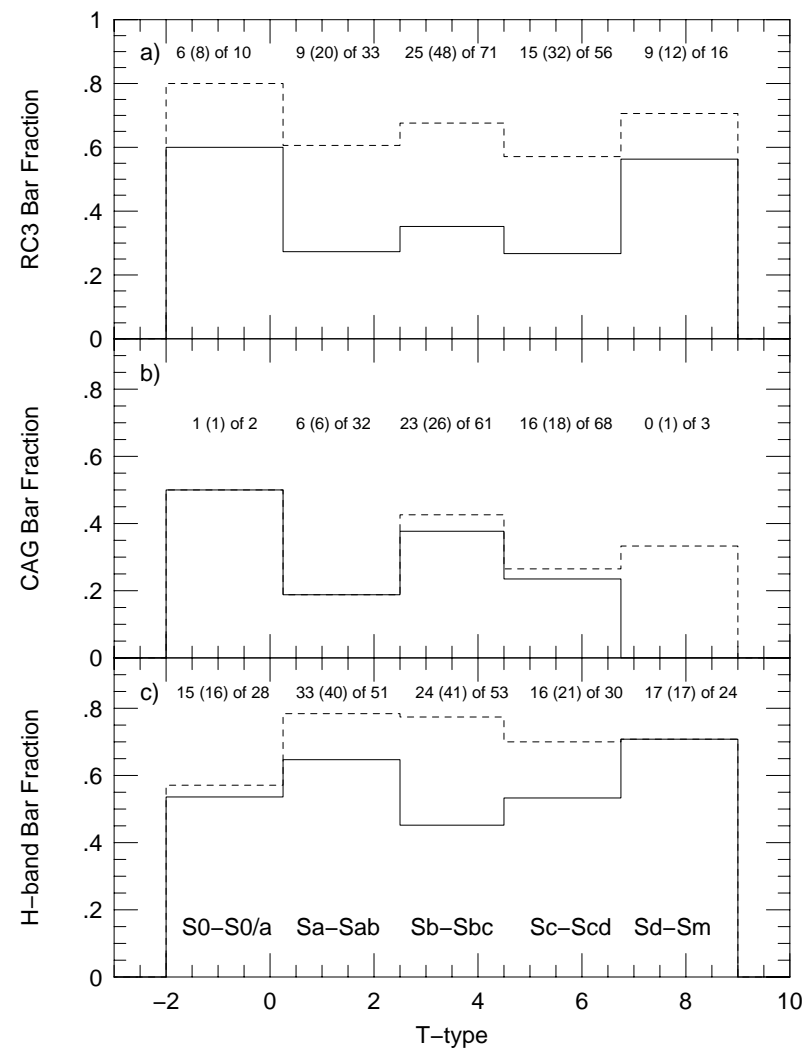

FIG. 4.-Bar-fraction as a function of morphological type: (a) optical types and bar classification from the RC3; (b) optical types and bar classification from the CAG; (c) $H$-band types and bar classification. The solid lines show the fraction of strong bars (SB). The dashed lines show the results of including weak bars (SAB or S/SB). The numbers above the histogram bins are the number of barred galaxies in that bin (including the weakly barred galaxies in parentheses) out of the total number of galaxies in that bin.

to be either strongly or weakly barred. Thus, the major difference between our $H$-band classification and the RC3 optical classification is that we find a much larger fraction of the sample to be strongly barred. Only $\sim 20 \%$ of the galaxies classified as unbarred (SA) in the $\mathrm{RC} 3$ have strong (SB) $H$-band bars; this is not a function of morphology. Roughly $40 \%-50 \%$ of galaxies classified as unbarred (SA) in the RC3 have detectable bars (SAB and SB) in the $H$-band. This fraction increases from $\sim 30 \%$ for $\mathrm{Sa}-\mathrm{Sab}$ galaxies up to $\sim 55 \%$ for $\mathrm{Sc}-\mathrm{Scd}$ galaxies. We speculate that this morphological effect is the result of the increased influence of dust and young stars on the optical morphology of late-type spirals compared with that of earlier types.

The CAG gives classifications for 166 of the galaxies in our sample. Of these, it classifies $27 \%$ as strongly barred and $31 \%$ as barred at any level. Thus, compared to the CAG, we find a much higher fraction of galaxies with bars. This is mainly because of the tendency of the CAG to classify galaxies with weak optical bars as unbarred. Because of this, the fraction of galaxies that are classified as unbarred in the CAG for which we find $H$-band bars is much larger than for the RC3. Nearly $50 \%$ of galaxies that are classified as unbarred in the CAG are strongly barred in the $H$ band. This number climbs to $\sim 60 \%$ when we include weak $H$-band bars. We find no evidence for any dependence on morphology in the CAG hidden bars.

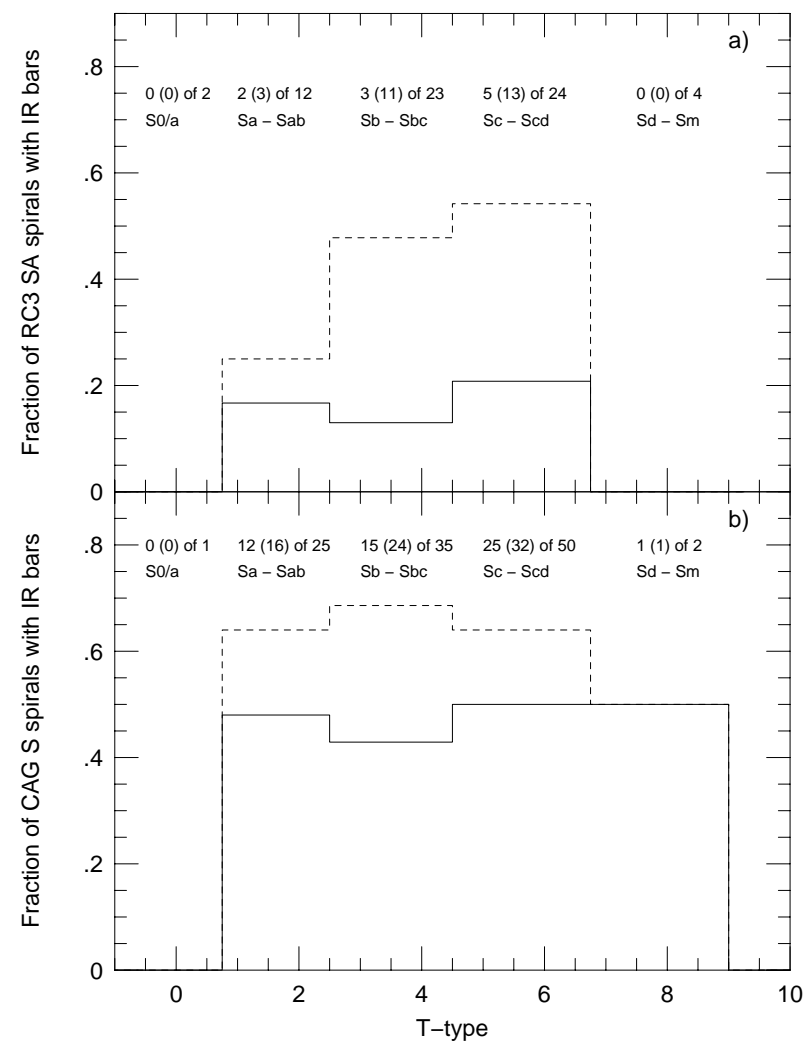

FIG. 5.-Fraction of optically unbarred galaxies with infrared bars plotted as a function of morphology: (a) optical types and bar classification from the RC3; (b) optical types and bar classification from the CAG. The solid lines show the fraction of strong bars (SB). The dashed lines show the results including weak bars (SAB or $\mathrm{S} / \mathrm{SB}$ ). The numbers above the histogram bins have the same meaning as in Fig. 4.

Although there are galaxies with optically hidden bars, visible only in the NIR, we disagree with claims found in the recent literature that all or nearly all bright spirals are barred in the NIR. The most complete recent study that comes to this conclusion is that of Seigar \& James (1998), who found a bar fraction of $90 \%$ in a nonstatistical sample of spiral galaxies. From this they argue that "this indicates that most, and quite possibly all, bright spirals are barred at some level." Clearly, we disagree, as we find no evidence for a bar in nearly $30 \%$ of our sample. It is tempting to ascribe the difference between our conclusion and theirs to sample differences; our sample is both larger and statistically better defined. However, there are other differences between the two studies that are worth consideration. First, our data are typically 1 mag deeper in surface brightness than are those of Seigar \& James (1998). Our observing times are comparable to theirs; they used the $3.8 \mathrm{~m}$ United Kingdom Infrared Telescope, with a pixel size of 0 ".29; our data were obtained mostly with a $1.8 \mathrm{~m}$ telescope and a detector with 1".5 pixels. As we are concerned with extended objects, the surfacebrightness limit scales linearly with both the aperture and the pixel size. Thus our images will typically probe a factor of $\approx 2.5$, or about 1 magnitude, deeper in surface brightness. On this basis, we should be more sensitive to the presence of bars than are Seigar \& James (1998). Second, Seigar \& James consider any oval distortion of the central region of a galaxy to be a bar. Third, they attempt full profile decompositions rather than our "classic," qualitative method. As a result, they may be more sensitive to weak bars. However, 


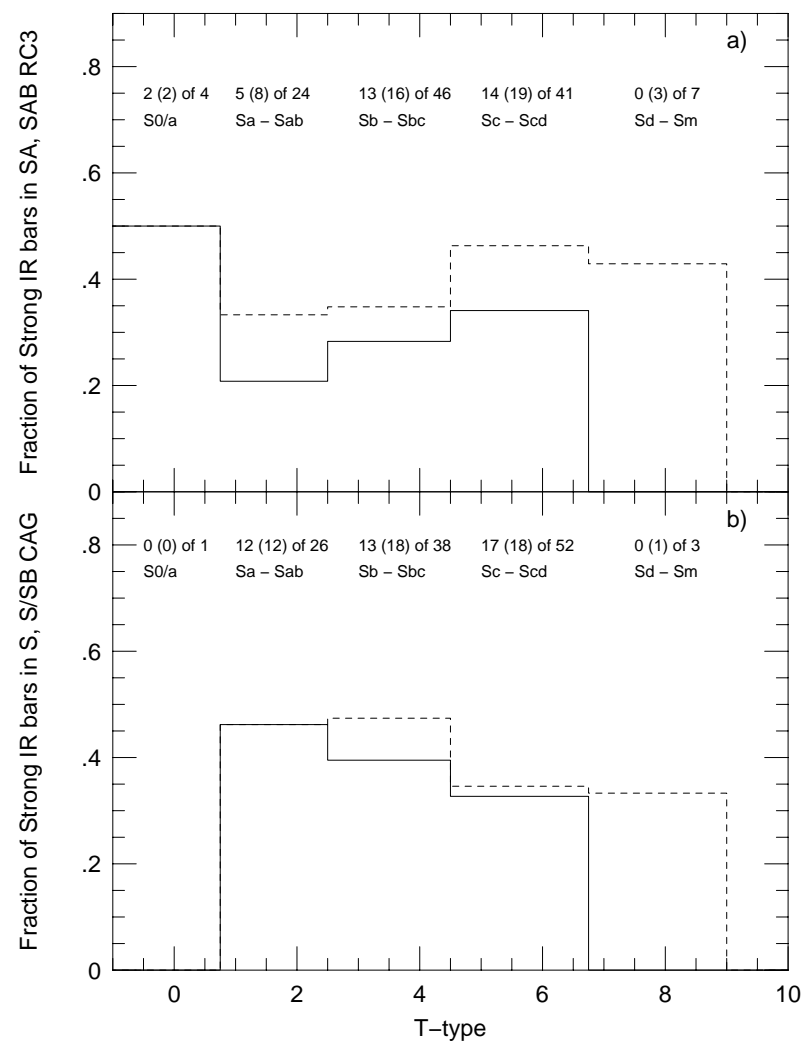

FIG. 6.-Fraction of galaxies not classified as strongly barred in the optical, but that are strongly barred in the $H$ band, plotted as a function of morphology: (a) optical types and bar classification from the RC3; (b) optical types and bar classification from the CAG. The solid lines show the fraction of galaxies that are optically unbarred (SA or S). The dashed lines show the results, including weak bars (SAB or $\mathrm{S} / \mathrm{SB})$. The numbers above the histogram bins have the same meaning as in Fig. 4.

the well-known failures of profile-fitting algorithms to find disks in some S0s suggest some caution on this point (see, e.g., Michard \& Marchal 1994). Finally, because they subtract models for the bulge and disk light before looking for bars, their method is very sensitive to how well the disk and bulge light distributions were modeled. For these reasons, we do not find the differences in the results of the two studies surprising. We believe that our results provide the most representative sample currently available of the bar fraction of galaxies in the nearby universe.

We find that late-type spirals ( $\mathrm{Sc}-\mathrm{Sm}$ ) have essentially the same bar fraction as early-type spirals $(\mathrm{Sa}-\mathrm{Sb})$. This is consistent with recent results on disk shape as a function of Hubble type (Rudnick \& Rix 1998; Zaritsky \& Rix 1997), arguing that both bars and disk asymmetries are due to the dynamical properties of disks. However, one might expect that bar lifetimes in the late-type spirals would be shorter than in early-type spirals for at least two reasons. First, as the disks of later type spirals have a much higher gas fraction than do those of earlier type spirals, angular momentum transfer should operate more strongly in later type spirals. Second, dark halos appear to dominate the dynamics of later type spirals (e.g., Courteau \& Rix 1999) much more than they do earlier type spirals (e.g., Moriondo, Giovanardi, \& Hunt 1998).

Dynamical models of disk galaxies indicate that bars are transient phenomena and can be induced by external interactions (e.g., Byrd \& Valtonen 1990; Gerin, Combes, \&
Athanassoula 1990). This suggests that the bar fraction of field and cluster spirals could be different. Although optical studies (e.g., Andersen 1996; Elmegreen, Elmegreen, \& Bellin 1990) find no compelling evidence for any such difference, it is worth examining our sample to see if any difference arises in the NIR. Given the magnitude limit of our survey, the only clusters represented are Fornax and Virgo. There are a total of 19 galaxies in our sample that are members of these clusters according to Ferguson (1989) and Binggeli, Sandage, \& Tammann (1985). Of these, we classify $12(63 \%)$ as barred, three $(16 \%)$ as weakly barred, and four $(21 \%)$ as unbarred. Thus we find that the fraction of barred galaxies in the Fornax and Virgo clusters is slightly higher than for our full sample, but the total numbers are small enough that this is not statistically significant.

A number of recent studies have attempted to evaluate the evolution of the distribution of Hubble types with increasing redshift (e.g., Lilly et al. 1998; Driver et al. 1998; Simard et al. 1999), but these studies generally avoid the issue of bars. Recently, Abraham et al. (1999) have claimed that the fraction of barred galaxies decreases with increasing redshift, becoming nearly zero beyond a redshift of $z \approx 0.5$. This apparent lack of bars at high redshift may be a crucial piece of information for understanding galaxy evolution. However, this result may be driven by a combination of systematics, involving both the bandpass shift of the rest frame with increasing redshift and the younger stellar populations in galaxy disks at increasing redshifts. In keeping with this, Bunker (1999) points out that several optically unbarred galaxies in the Hubble Deep Field (HDF) reveal bars in the NICMOS HDF data. Although Abraham et al. claim that the bandpass effect is insufficient to account for their result, it may be that the combination of the shift to bluer rest wavelengths and the younger mean stellar population of galaxy disks at look-back times of 4-7 Gyr is sufficient to account for the lower detection frequency of bars in the Abraham et al. (1999) sample. A careful modeling study of these effects should shed considerable light on this issue.

In our next paper, we will present a full discussion of the optical and NIR morphologies of the galaxies in our sample, based on our $B$ - and $H$-band images. This will allow us to consider the strength of features such as rings as a function of wavelength. Returning to the specific issue of bars, we note that "bar strength" does not map onto a single physical variable (at least two are involved: relative flux at a given wavelength and bar axial ratio). A number of different quantitative measures of "bar strength " have been proposed in the literature (e.g., Elmegreen \& Elmegreen 1985; Ohta, Hamabe, \& Wakamatsu 1990; Martin 1995; Wozniak et al. 1995; Abraham et al. 1999), all of which measure different things. We will apply a collection of these various measures to our sample in an attempt to put the phrase "bar strength" on a firm, consistent, quantitative footing. As part of this study, we also plan to examine the isophotal shapes of bulges in barred and unbarred spirals.

We thank the many OSU graduate students who collected data for this project, and we wish to note especially the many nights of work that Ray Bertram and Mark Wagner have devoted to the OSU survey. We are grateful to Roberto Aviles for obtaining many of the optical images of southern galaxies for us with the $0.9 \mathrm{~m}$ telescope at the Cerro Tololo Inter-American Observatory (CTIO). We 
thank Bob Williams and Malcolm Smith, past and present directors of CTIO, for the generous allotment of telescope time needed to observe most of our southern sample. J. A. F. thanks Leonard Searle for the observing opportunities provided by a Visiting Research Associateship at Las Campanas Observatory, where some of these data were obtained. We are pleased to thank Ron Buta and Allan
Sandage for several very useful discussions on galaxy morphology and Roelof deJong for his comments. J. A. F. acknowledges support from a PPARC Senior Visiting Research Fellowship (grant GR/L00896) held in 1996 at the University of Durham Department of Physics. This work was supported by grants AST 92-17716 and AST 96-17006 from the National Science Foundation.
Abraham, R. G., Merrifield, M. R., Ellis, R. S., Tanvir, N. R., \& Brinchmann, J. 1999, MNRAS, 308, 569

Andersen, V. 1996, AJ, 111, 1805

Binggeli, B., Sandage, A., \& Tammann, G. A. 1985, AJ, 90, 1681

Block, D. L., Bertin, G., Stockton, A., Grosbol, P., Moorwood, A. F. M., \& Peletier, R. F. 1994, A\&A, 288, 365

Block, D. L., \& Wainscoat, R. J. 1991, Nature, 353, 48

Bosma, A. 1996, in ASP Conf. Ser. 91, Barred Galaxies, ed. R. Buta, D. Crocker, \& B. G. Elmegreen (San Francisco: ASP), 132

Bottema, R. 1993, A\&A, 275, 16

Bunker, A. J. 1999, in ASP Conf. Ser. 191, Photometric Redshifts and High

Redshift Galaxies, ed. R. Weymann, L. Storrie-Lombardi, M. Sawicki, \&

R. Brunner (San Francisco: ASP)

Buta, R. 1986, ApJS, 61, 609 1995, ApJS, 96, 39

Byrd, G., \& Valtonen, M. 1990, ApJ, 350, 89

Combes, F., Debbasch, F., Friedli, D., \& Pfenniger, D. 1990, A\&A, 233, 82

Courteau, S., \& Rix, H.-W. 1999, ApJ, 513, 561

Debattista, V. P., \& Sellwood, J. A. 1998, ApJ, 493, L5

de Vaucouleurs, G. 1963, ApJS, 8, 31

de Vaucouleurs, G., de Vaucouleurs, A., Corwin, H. G., Jr., Buta, R. J.,

Paturel, G., \& Fouqué, P. 1991, Third Reference Catalogue of Bright Galaxies (New York: Springer) (RC3)

Driver, S. P., Fernandez-Soto, A., Couch, W. J., Odewahn, S. C., Wind-

horst, R. A., Phillips, S., Lanzetta, K., \& Yahil, A. 1998, ApJ, 496, L93

Elmegreen, B. G., \& Elmegreen, D. M. 1985, ApJ, 288, 438

Elmegreen, D. M., Elmegreen, B. G., \& Bellin, A. 1990, ApJ, 364, 415

Eskridge, P. B., et al. 2000, in preparation

Ferguson, H. C. 1989, AJ, 98, 367

Frogel, J. A., Quillen, A. C., \& Pogge, R. W. 1996, in New Extragalactic Perspectives in the New South Africa, ed. D. Block \& J. M. Greenberg (Dordrecht: Kluwer), 65

Gerin, M., Combes, F., \& Athanassoula, E. 1990, A\&A, 230, 37

Hackwell, J. A., \& Schweizer, F. 1983, ApJ, 265, 643
Knapen, J. H., Shlosman, I., \& Peletier, R. F. 2000, ApJ, in press

Kormendy, J. 1982a, in Morphology and Dynamics of Galaxies, ed. L. Martinet \& M. Mayor (Sauverny: Geneva Obs.), 115 .1982b, ApJ, 257, 75

Kuijken, K., \& Merrifield, M. R. 1995, ApJ, 443, L13

Lilly, S., et al. 1998, ApJ, 500, 75

Lindblad, P. A. B., Lindblad, P. O., \& Athanassoula, E. 1996, A\&A, 313, 65

Martin, P. 1995, AJ, 109, 2428

Michard, R., \& Marchal, J. 1994, A\&AS, 105, 481

Moriondo, G., Giovanardi, C., \& Hunt, L. K. 1998, A\&A, 339, 409

Mulchaey, J., \& Regan, M. 1997, ApJ, 482, L135

Naim, A., et al. 1995, MNRAS, 274, 1107

Ohta, K., Hamabe, M., \& Wakamatsu, K.-I. 1990, ApJ, 357, 71

Pfenniger, D., \& Norman, C. 1990, ApJ, 363, 391

Piner, B. G., Stone, J. M., \& Teuben, P. J. 1995, ApJ, 449, 508

Quillen, A. C., \& Frogel, J. A. 1997, ApJ, 487, 603

Quillen, A. C., Frogel, J. A., Pogge, R. W., DePoy, D. L., \& Kenney, J. D. 1995, ApJ, 441, 539

Rudnick, G., \& Rix, H.-W. 1998, AJ, 116, 1163

Sandage, A., \& Bedke, J. 1994, The Carnegie Atlas of Galaxies (Washington: Carnegie Inst.) (CAG)

Seigar, M. S., \& James, P. A. 1998, MNRAS, 299, 672

Sellwood, J. A., \& Wilkinson, A. 1993, Rep. Prog. Phys., 56, 173

Shlosman, I., Frank, J., \& Begelman, M. C. 1989, Nature, 338, 45

Simard, L., et al. 1999, ApJ, 519, 563

Thronson, H. A., Jr., Hereld, M., Majewski, S., Greenhouse, M., Johnson, P., Spillar, E., Woodward, C. E., Harper, D. A., \& Rauscher, B. J. 1989, ApJ, 343, 158

Toomre, A. 1969, ApJ, 158, 899

Weiner, B. J., Sellwood, J. A., Williams, T. B., \& van Gorkom, J. H. 1996, BAAS, 28, 1344

Wozniak, H., Friedli, D., Martinet, L., Martin, P., \& Bratschi, P. 1995, A\&AS, 111, 115

Zaritsky, D., \& Rix, H.-W. 1997, ApJ, 477, 118 